\title{
SULLA MISURA DELL'ASSORBIMENTO IONOSFERICO
}

\author{
Piero Dominici
}

1. - E ben nota la grande importanza che hanno assunto, sia nel campo scientifico, sia nel campo pratico, le questioni riguardanti la ionosfera. Dal lontano 1902, quando, Kennelly, Heaviside, e Nagaoka ipotizzarono l'esistenza nell'alta atmosfera di regioni fortemente ionizzate e dotate di potere riflettente per le radioonde, gli studi ionosferici sono stati sempre più sviluppati, ed ai nostri giorni si può dire che non vi è paese civile in cui non ci si dedichi con grande interesse a queste ricerche.

Parallelamente allo sviluppo degli studi teorici, veramente cospicuo è stato anche il progredire ed il raffinarsi dei metodi sperimentali. Appleton, nel 1925, con una celebre esperienza, metteva in evidenza, per la prima volta, l'esistenza della ionosfera, ed al tempo stesso indicava il primo metodo di studio sperimentale della stessa. Il successivo metodo dovuto a Breit e Tuve, basato sui sondaggi verticali delle regioni ionosferiche a mezzo di brevi treni d'onda (metodo degli impulsi), ha permesso, col continuo progredire della tecnica elettronica, la costruzione delle moderne ionosonde, apparati di grande efficienza, di notevole sicurezza ed a funzionamento completamente automatico.

E noto che la presenza di elettroni liberi nella ionosfera porta ad una modificazione dell'indice di rifrazione, che, trascurando l'azione del campo geomagnetico, vale:

$$
n=\sqrt{1-\frac{N e^{2}}{\pi m f^{2}}}
$$

dove $e, m$ sono la carica e la massa dell'elettrone, $N$ la densità elettronica, $f$ la frequenza dell'onda. Se la frequenza dell'onda emessa ad impulsi è tale da annullare $n$, in rapporto alla densità elettronica di un certo strato ionosferico, cioè se è $f=\sqrt{\frac{e^{\tau} N}{\pi m}}$ si ha la riflessione dell'onda sullo strato, e gli impulsi tornano verso la terra. Qui ven- 
gono captati da un ricevitore sintonizzato col trasmettitore, poi rivelati ed applicati al registratore, che in genere è un tubo a raggi catodici di cui si fotografa convenientemente lo schermo. Il tempo intercorso fra l'emissione di un impulso e la ricezione della sua eco, ricavabile con una tecnica particolare del sistema registratore, ci dà l'altezza rispetto al suolo dello strato che ha riflesso il segnale. Questa altezza, ricavata supponendo che l'onda viaggi nella ionosfera ad una velocità costante pari a quella della luce nel vuoto - il che non ̀̀ - si chiama "altezza virtuale » dello strato ed è maggiore dell'altezza vera. Aumentando la frequenza dell'onda, lo strato ad un certo momento cessa di essere riflettente, e l'onda lo attraversa. Il valore della frequenza massima ancora riflessa si chiama "frequenza critica " dello strato, ed in corrispondenza si ha il valore massimo della densita elettronica in quel particolare strato, pari a $N_{0}=1,26 \cdot 10^{4}(f)^{2}{ }_{\mathrm{mhz}}$ elettroni $/ \mathrm{cm}^{3}$.

In genere il campo di frequenza delle ionosonde comunemente adoperate negli osservatori ionosferici è da 1 a $20 \mathrm{Mhz}$ ca., e questo per la difficoltà di generare e ricevere impulsi di minore frequenza. Ciò significa che le ionosonde sono atte ad esplorare regioni ionosferiche con densità elettronica non minore di circa $2.10^{4}$ elettroni $/ \mathrm{cm}^{3}$, vale a dire gli strati $E$ (quota circa $100 \mathrm{~km}$ ) ed $F$ (quota fra 200 e $400 \mathrm{~km}$ ), e non possono dirci nulla, almeno con sicurezza, delle regioni inferiori (strato $D$ ). In realtà gli strati $E$ ed $F$, particolarmente questo ultimo sono gli strati decisamente più interessanti, e quelli che determinano le condizioni della radiopropagazione a grande distanza, tanto che, sinché si sono riguardati solo problemi di propagazione delle onde corte, è stata del tutto trascurata l'importanza di un eventuale o di eventuali strati a più hassa quota. Questa posizione si è dovuta però rivedere non appena si è voluto affrontare il problema del calcolo dell'intensità di campo a grande distanza.

$\mathrm{Si}$ sa che un'onda propagandosi nella ionosfera si attenua. Se l'intensità di campo era $E_{0}$ in partenza, all'arrivo, dopo un certo tragitto nella ionosfera, essa varrà $E_{0} \mathrm{e}^{-\mathrm{l}}$, e $\Gamma$ si chiama assorbimento ionosferico relativo a quel certo tragitto. In una recente memoria ( $\left.{ }^{1}\right)$ il prof. Bolle ed io abbiamo trattato, limitandoci al campo delle onde corte, la questione del calcolo dell'assorbimento ionosferico. Abliamo dimostrato che per le onde corte si può ritenere, dal punto di vista dell'assorbimento, che il campo geomagnetico non ha apprezzabile

(1) Annali di Geofisica, vol. V, n. 3, 1952. 
influenza, ed abbiamo poi ricavato un serie di formule che danno l'assorbimento strato per strato, ed a seconda che l'onda si rifletta sullo strato o lo attraversi. In queste formule, che, essendo del tutto teoriche, dovranno poi avere la controprova dell'esperienza, compaiono come parametri due valori caratteristici dell'onda, cioè la frequenza e l'inclinazione del raggio considerato, e due caratteristici dello strato, cioc̀ la frequenza delle collisioni fra elettroni liberi e molecole neutre (legata alla pressione) e la massima densita elettronica (legata all'agente ionizzante, cioè alla radiazione solare). Facciamo un esempio tipico: un'onda che si rifletta sullo strato $F$ e che quindi attraversi lo strato $E$ e lo strato $D$. L'assorbimento complessivo $\Gamma$ sarà la somma dell'assorbimento in $F, \Gamma_{0 F}$ (assorbimento della regione deviatrice), più l'assorbimento in $E$ ed in $D, \Gamma_{1 E}+\Gamma_{1 D}$ (assorbimento nelle regioni non deviatrici). Ora, l'assorbimento complessivo $\Gamma$ si può misurare con uno dei metodi che descriverò più avanti, e, come bo delto, gli assorbimenti relativi agli strati $E$ e $F$ si possono calcolare a partire dai dati forniti dalle ionosonde. Si può isolare cosi il termine competente dell'assorbimento dello strato $D$, e da questo risalire alle caratteristiche dello strato stesso. Ciò è di grande aiuto per la previsione dell'intensità di campo all'arrivo nelle comunicazioni a grande distanza.

Veramente le caratteristiche dello strato $D$ si possono ricavare anche con la tecnica dei sondaggi ad impulsi, adoperando apposite apparecchiature, alquanto diverse dalle comuni ionosonde, ma comunque facilmente realizzabili nei nostri laboratori. Il motivo principale che ha determinato l'inserimento delle misure sistematiche dell'assorbimento ionosferico nel piano di lavoro del gruppo ionosferico dell'Istituto Nazionale di Geofisica è la straordinaria utilità di quelle misure per lo studio delle relazioni tra fenomeni ionosferici e fenomeni geomagnetici e solari. Mi limito a ricordare la stretta connessione già da tempo rilevata fra le perturbazioni geomagnetiche ad inizio rapido e le evanescenze brusche delle radio comunicazioni, e fra la comparsa di macchie solari e l'irregolare andamento della ionosfera. Questi sono due esempi tipici di questioni che sono difficilmente studiabili sperimentalmente con le normali ionosonde, ma che si possono rilevare con grande comodità dall'andamento dell'assorbimento ionosferico.

2. - Esaminerò ora brevemente $\mathrm{i}$ metodi comunemente impiegati per la misura dell'assorbimento ionosferico. 
Il primo, e più antico, che chiamerò "metodo ad onda continua ", fu usato la prima volta da Appleton nel 1930. Questo metodo si basa sul ben noto fenomeno dell'evanescenza (fading), dovuto all'interferenza fra onda diretta e onda spaziale.

Una stazione sita in $A$ (fig. 1) emette delle radioonde di frequenza costante le quali giungono al posto ricevente $C-$ a poche decine di chilometri di distanza - seguendo in parte il percorso $A C$ (onda diretta) ed in parte il percorso $A B C$ (onda spaziale) con riflessione in $B$ sulla ionosfera. Sull'antenna del ricevitore sono presenti contemporaneamente segnali dovuti alle due onde, diretta e spaziale;

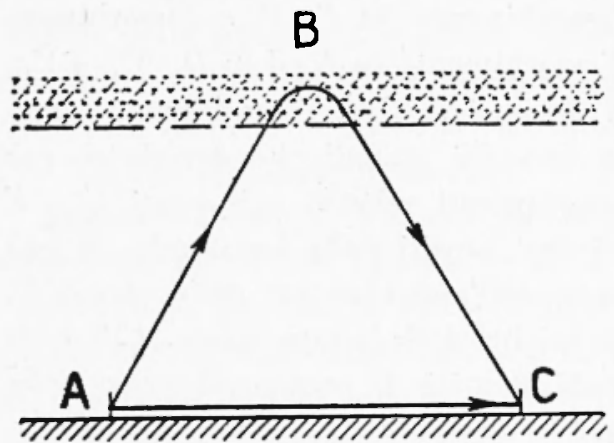

Fig. 1 quest'ultima è variamente atte. nuata lungo il percorso ionosferico ed ha una certa differenza di fase $\Psi$, variabile nel tempo, rispetto all'onda diretta. Il ricevitore è provvisto di un misuratore o registratore di uscita accuratamente tarato in microvolt/metro della tensione a radiofrequenza all'antenna. Se la ionosfera fosse un riflettore perfetto, l'intensità di campo $E_{1}$ dell'onda spaziale in $C$ sareblue solo inversamente proporzionale alla distanza percorsa $d_{1}$ secondo una costante $k$ dipendente solo dalla potenza effettivamente irradiata dall'antenna trasmittente. In realtà lo strato non è un riflettore perfetto, ma ha un coefficiente di riflessione $\varrho$ minore dell'unità. Osservo subito che $\varrho$ è legato all'assorbimento $\Gamma$ dalla relazione $\Gamma=-\log \theta$.

Si ha

$$
E_{1}=\frac{k}{a_{1}} \varrho \text {, ciò̀ : } \varrho=\frac{d_{1}}{k} E_{\mathrm{t}}=\frac{d_{1}}{k} E_{\mathrm{o}} \begin{aligned}
& E_{1} \\
& F_{\mathrm{o}}
\end{aligned},
$$

dove $E_{o}$ è l'intensità di campo dell'onda diretta, facilmente misurabile eliminando in un modo o nell'altro $E_{1}$, ad esempio, come fece Appleton, misurandola di notte, quando cioè, nelle condizioni della sua esperienza $(\hat{\lambda}=491 \mathrm{~m})$ mancava l'onda spaziale. Il rapporto $E_{1} / E_{0}$ si ricava osservando $i$ massimi ed i minimi della curva del registratore di uscita. Infatti l'intensità di campo all'aereo ricevente è :

$$
E^{2}=E_{,}^{2}+E_{1}^{2}+2 E_{\mathrm{o}} E_{1} \operatorname{css} \varphi
$$


cioè, per $\uparrow=O, E_{\mathrm{M}}=E_{\mathrm{o}}+E_{\mathrm{I}}$ (valore massimo), per $\varphi=\pi$, $E_{\mathrm{m}}-E_{\mathrm{o}}-E_{1}$ (valore minimo) si ha così

$$
\frac{E_{1}}{F_{0}}=\frac{F_{\mathrm{M}}-E_{\mathrm{m}}}{F_{\mathrm{M}}+F_{\mathrm{m}}}
$$

Noto che la $E_{1}$ non si mantiene costante ma varia a sua volta seguendo le variazioni della ionizzazione. Tuttavia è facile distinguere da eventuali evanescenze da assorbimento le evanescenze di fase utili per la determinazione del rapporto $E_{1} / E_{0}$, sia per la loro maggiore appariscenza, sia per il loro caratteristico andamento regolare. Conosciuto il rapporto $E_{1} / E_{0}$, nota $E_{0}$ assunto per $d_{1}$ il valore $2 h^{\prime}$, dove $h^{\prime}$ è l'altezza dello strato riflettente - cosa lecita se trasmettitore e ricevitore non sono molto lontani -, calcolata $k$ in base alle caratteristiche della stazione trasmittente, rimane determinato $\varrho$ o, che è lo stesso, I'.

Una causa di gravi errori in questo metodo è costituita da eventuali variazioni della potenza emessa dal trasmettitore o da variazioni nella sintonia tra trasmettitore e ricevitore. Precauzioni comunemente usate nelle moderne apparecchiature di questo tipo - un esempio ne è l'apparato del National Bureau of Standards di Washington $\left({ }^{2}\right)$ consistono nell'uso di tensioni di alimentazione assolutamente stabilizzate e nell'uso di cristalli di quarzo per il controllo della frequenza sia nel trasmettitore che nel ricevitore.

Un secondo metodo è quello "ad impulsi », derivato dalla omonima tecnica di sondaggio ionosferico.

Anche qui un trasmettitore modulato ad impulsi lancia verso l'alto dei brevi treni d'onda di una certa frequenza e gli echi riflessi dalla ionosfera vengono captati da un ricevitore sito po-

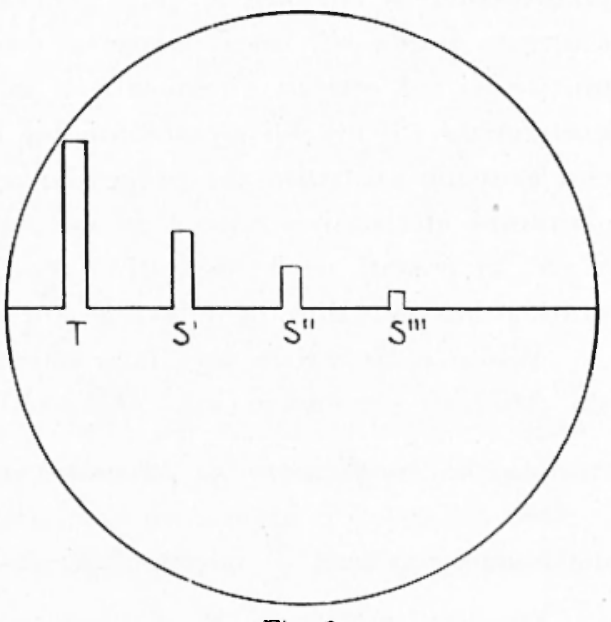

Fig. 2 co lontano, ed applicati ad un tubo a raggi catociici, ii cui asse aei tempi è sincronizzato con la cadenza degli impulsi. Sullo schermo del tubo apparirì una traccia sul tipo di quella schematizzata nella fig. 2,

(2) N.B.S. - circolare n. 462 - Washington, 1948. 
in cui si notano il segnale dovuto all'impulso di partenza $T$, e quelli $S^{\prime} S^{\prime \prime} S^{\prime \prime \prime}$ dovuti al primo secondo terzo eco ecc. ecc.

Riferendoci alla fig. 1 , seguitiamo a chiamare $d_{0}$ la distanza fra trasmettitore e ricevitore e $d_{i}$, al solito preso uguale al doppio dell'altezza dello strato, la lunghezza del tragitto dell'onda spaziale. $T, S^{\prime}, S^{\prime \prime}$ ecc. siano le misure in mun dell'altezza del segnale dovuto al segnale diretto, al primo eco ecc.

E evidentemente $T=\frac{\alpha}{d_{\mathrm{o}}}$, dove $\alpha$ è una costante. Quanto ad $S^{\prime}$, ̀̀ $S^{\prime}=\frac{\beta}{d_{1}} \varrho$, dove $\beta$ è un'altra costante. Così per $S^{\prime \prime}=\frac{\beta}{2 d_{1}} \varrho^{2}$, avendo preso uguale all'unità il coefficiente di riflessione del suolo terrestre. Si ha allora:

$$
\varrho=2 \frac{S^{\prime \prime}}{S^{\prime}}
$$

Il rapporto $S^{\prime \prime} / S^{\prime}$ si misura direttamente sullo schermo dell'oscillografo e e è conosciuto. In pratica la cosa non è così semplice. Anche ammesso che la natura del suolo sia tale da non influire apprezzabilmente sulla riflessione dell'onda, rimane pur tuttavia la difficoltà grandissima di rilevare $S^{\prime \prime}$, che generalmente è piuttosto debole, dall'intenso fondo di interferenze e disturbi che affetta normalmente misure di tal genere. Preziosa è a tal riguardo l'esperienza fatta recentemente (1950) dai ricercatori del Bureau lonosphérique Francais, che avevano costruito un pregevole apparato ad impulsi con un registratore elettronico capace di calcolare automaticamente il rapporto $S^{\prime \prime} / S^{\prime}$. Si è stati però costretti a modificare l'apparato a causa della pratica impossibilità di rilevare con sicurezza l'eco $S^{\prime \prime}$.

White e Brown in una loro esperienza del 1935, aggirarono questa difficoltà servendosi solo dell'eco di prima riflessione. Infatti, introdotto il coefficiente di trasmissione $K=\frac{\alpha}{\beta d_{0}}$, si ha $\varrho=K d_{1} \frac{S^{\prime}}{T}$; ma è anche $\varrho=2 \frac{S^{\prime \prime}}{S^{\prime}}$, quindi $K=\frac{2}{d_{1}} \frac{\varsigma^{\prime \prime}}{S^{\prime}} \frac{T}{S^{\prime}}$

Questo coefficiente $K$ naturalmente si determina una volta per tutte, e quindi per la misura si opera solo con $T$ ed $S^{\prime}$, che sono segnali sicuri e facilmente rilevabili.

3. - Il metodo ad impulsi è senz'altro più completo e preciso che non il metodo ad onda continua, innanzi tutto perché in pratica è esente da interferenze dovute ad emissioni di altre stazioni sulla medesima 
frequenza, ed in secondo luogo perché permette di misurare separatamente l'assorljimento subito da ognuna delle due componenti magneto-ioniche dell'onda, cosa quest'ultima che, a meno di delicati artifici, non si può ottenere col metodo ad onda continua.

Tuttavia l'esigenza che si è di più cercato di soddisfare nella esecuzione delle nostre misure, è la registrazione dei dati, e mi pare clıc, dei due metodi, quello più efficiente e comodo da questo punto

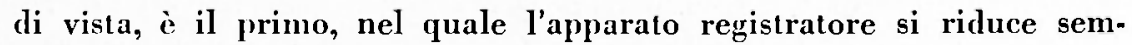
plicemente ad un volimetro scrivente od a registrazione fotografica, convenientemente applicato al circuito del controllo automatico di sensibililà del ricevitore.

II progetto di massima per l'esecuzione delle nostre misure di assorbimento $i$, salvo varianti imposte da imprevedute necessità, il seguente.

Nell'osservatorio di S. Alessio dell'I.N.G., presso Roma, sarà istallato un trasmettitore di moderata potenza ad onda non modulata di frequenza sui $3 \mathrm{Mhz}$, con controllo a quarzo della frequenza emessa. Poco lontano, sempre nell'ambito dell'Osservatorio, un semplice registratore di intensità di campo di piccola sensibilita fornirà la quantiti $E_{0}$. Un vantaggio notevole nella introduzione di questo primo registratore è di svincolarsi dalla necessità dell'assoluta costanza della potenza emessa: questa condizione non è completamente raggiungibile neanche con la integrale stabilizzazione delle tensioni di alimentazione - cosa già abluastanza difficile - a causa dell'eventuale deperimento dei tubi o di altri componenti dei circuiti. La $E_{1}$ invece sarà misurata nell'Osservatorio dell'I.N.G. di Rocca di Papa, a $10 \mathrm{~km} \mathrm{ca.}$ in linea d'aria, mediante un sensibile ricevitore accordato seguito dal complesso voltmetrico di registrazione. Le antenne probabilmente saramno a telaio per separare meglio eventuali interferenze. Ogni 10 minuti il trasmettitore cesserà di emettere per un breve tempo e ogni ora per un tempo più lungo. Cosi, oltre ad avere sulle registrazioni una comune hase dei tempi, si potranno discernere dal segnale utile i sernali interferenti e i disturbi di varia natura. Il confronto fra $E_{1}$ e $E_{a,}$ fornirì $\Gamma$ al modo già visto, cioè $\Gamma=-\log \frac{E_{0}}{E_{1}}$.

Roma - Istiluto Nazionale di Geofisica - Giugno 1952. 


\section{RIASSUNTO}

Relativamente alla misura dell'assorbimento ionosferico, se ne illustra brevemente l'importanza ed il significato. V'engono quindi esaminati $i$ metodi di misura comunemente usati.

\section{SUMMARY}

Concerning the measurement of ionospheric absorption and a brief outline of its importance and significance. The measurement methods commonly used are examined and discussed. 